\title{
Duodenal amyloidosis secondary to ulcerative colitis
}

\author{
Seung Woon Park, Sam Ryong Jee, Ji Hyun Kim, Sang Heon Lee, Jin Won Hwang, Ji Geon Jang, \\ Dong Woo Lee, Sang Yong Seol \\ Department of Internal Medicine, Inje University Busan Paik Hospital, Inje University College of Medicine, Busan, Korea
}

Amyloidosis is defined as the extracellular deposition of non-branching fibrils composed of a variety of serum-protein precursors. Secondary amyloidosis is associated with several chronic inflammatory conditions, such as rheumatologic or intestinal diseases, familial Mediterranean fever, or chronic infectious diseases, such as tuberculosis. Although the association of amyloidosis with inflammatory bowel disease is known, amyloidosis secondary to ulcerative colitis (UC) is rare. A 36-year-old male patient with a 15-year history of UC presented with nausea, vomiting, and abdominal pain. He had been treated with infliximab for 6 years. At the time of admission, he had been undergoing treatment with mesalazine and adalimumab since the preceding 5 months. Esophagogastroduodenoscopy showed mucosal erythema, edema, and erosions with geographic ulcers at the 2nd and 3rd portions of the duodenum. Duodenal amyloidosis was diagnosed using polarized light microscopy and Congo red stain. Monoclonal gammopathy was not detected in serum and urine tests, while the serum free light chain assay result was not specific. An increase in plasma cells in the bone marrow was not found. Secondary amyloidosis due to UC was suspected. The symptoms were resolved after glucocorticoid therapy. (Intest Res 2018;16:151-154)

Key Words: Amyloidosis; Colitis, ulcerative; Duodenum

\section{INTRODUCTION}

Secondary (AA) amyloidosis is caused by extracellular deposition of fibrils that are composed of serum amyloid A (SAA) protein, an acute phase reactant. It can occur in association with many chronic inflammatory conditions, including rheumatoid arthritis, ankylosing spondylitis, IBD, and periodic fever syndromes or with chronic infections such as tuberculosis and osteomyelitis. ${ }^{1,2}$ AA amyloidosis develops in IBD patients and shows persistent, long-lasting, uncontrolled inflammatory activity. Although IBD is a known cause of AA amyloidosis, AA amyloidosis due to UC is extremely rare. ${ }^{3,4} \mathrm{~A}$ clinical presentation of renal impairment with proteinuria and nephrotic syndrome accounts for most of these cases, while the development of renal insufficiency is the

Received December 12, 2016. Revised February 26, 2017.

Accepted March 17, 2017. Published online August 2, 2017

Correspondence to Sam Ryong Jee, Department of Internal Medicine, Inje

University Busan Paik Hospital, Inje University College of Medicine, 75 Bokji-

ro, Busanjin-gu, Busan 47392, Korea. Tel: +82-51-890-6536, Fax: +82-51-

892-0273, E-mail: tokimom@nate.com most important predictor of mortality in amyloidosis. We report a case of duodenal amyloidosis with long-standing UC.

\section{CASE REPORT}

A 36-year-old male patient with a 15-year history of UC presented with nausea, vomiting, and abdominal pain. When he was first diagnosed with UC 15 years ago, colonoscopy showed multiple ulcers, mucosal friability, and bleeding throughout the entire colon and rectum. The Mayo score was 9. After being diagnosed with UC, he was treated with mesalazine and intermittent steroid therapy for 9 years, until cyclosporine therapy was administered due to development of steroid-refractory UC 3 years later. As cyclosporine was not effective, he was then treated with infliximab for 6 years. Currently, he is being treated with mesalazine and adalimumab since 5 months. Clinical tests revealed mucosal friability and multiple erosions without ulcers and bleeding throughout the entire colon, while the Mayo score was 4. Upon physical examination, a normoactive bowel sound was heard, the abdomen was found to be flat, and tender-

\footnotetext{
๑ Copyright 2018. Korean Association for the Study of Intestinal Diseases. All rights reserved.

This is an Open Access article distributed under the terms of the Creative Commons Attribution Non-Commercial License (http://creativecommons.org/licenses/by-nc/4.0)

which permits unrestricted non-commercial use, distribution, and reproduction in any medium, provided the original work is properly cited.
} 
ness was absent. No hepatomegaly or splenomegaly was noted. The laboratory results revealed leukocytosis (white

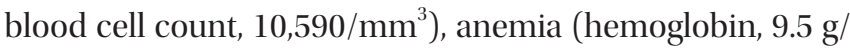
$\mathrm{dL}$ ), and an elevated CRP level of $5.38 \mathrm{mg} / \mathrm{dL}$. Esophagogastroduodenoscopy showed mucosal erythema, edema, and erosions with geographic ulcers at the 2nd and 3rd portions of the duodenum (Fig. 1). Biopsy of the duodenum and histological examination were performed. Light microscopy with $H \& E$ staining showed pink, amorphous hyaline material in the mucosa and submucosa of the duodenum (Fig. 2A). Polarized light microscopy using Congo red stain revealed amyloid deposits that exhibited characteristic apple-green birefringence (Fig. 2B). To distinguish between primary (AL) and AA amyloidosis, other tests, including serum and urine protein electrophoresis, immunofixation electrophoresis, serum free light chain (FLC) assay, and bone marrow biopsy, were performed. Monoclonal gammopathy was not detected in serum and urine tests, while the serum FLC assay result was not specific. An increase in the number of plasma cells in the bone marrow was not found. Excluding AL amyloidosis, AA amyloidosis due to UC was suspected. There was no evidence suggesting involvement of other organs. The serum creatinine level was normal $(0.73 \mathrm{mg} / \mathrm{dL})$ and 24 -hour urine protein excretion was $55.5 \mathrm{mg} /$ day. There were no abnormal findings on echocardiography. Methylprednisolone (60 mg/ day) was administered to control UC and therefore treat the duodenal amyloidosis. All symptoms resolved in a few days, and the patient was discharged with oral glucocorticoids. After 2 months of steroid treatment, follow-up esophagogastroduodenoscopy was performed, which indicated that the edema, erosion, and geographic ulcers of the duodenum were ameliorated (Fig. 3).

\section{DISCUSSION}

Amyloidosis is a general term used for disorders charac-
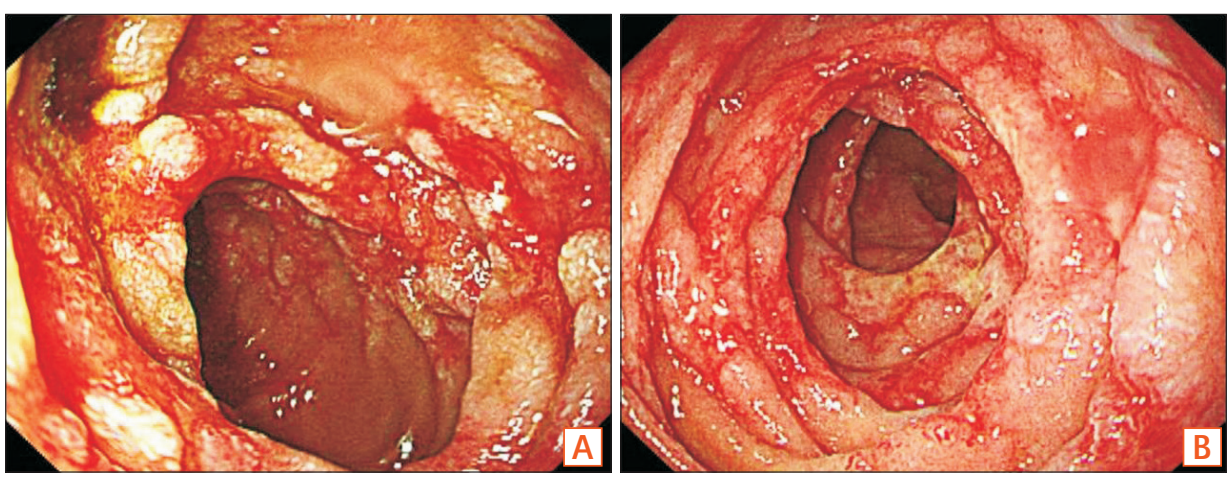

Fig. 1. Endoscopic findings. Mucosal edema, erythema, erosions, and geographic ulcers were observed at the (A) duodenal 2nd portion and (B) 3rd portion.
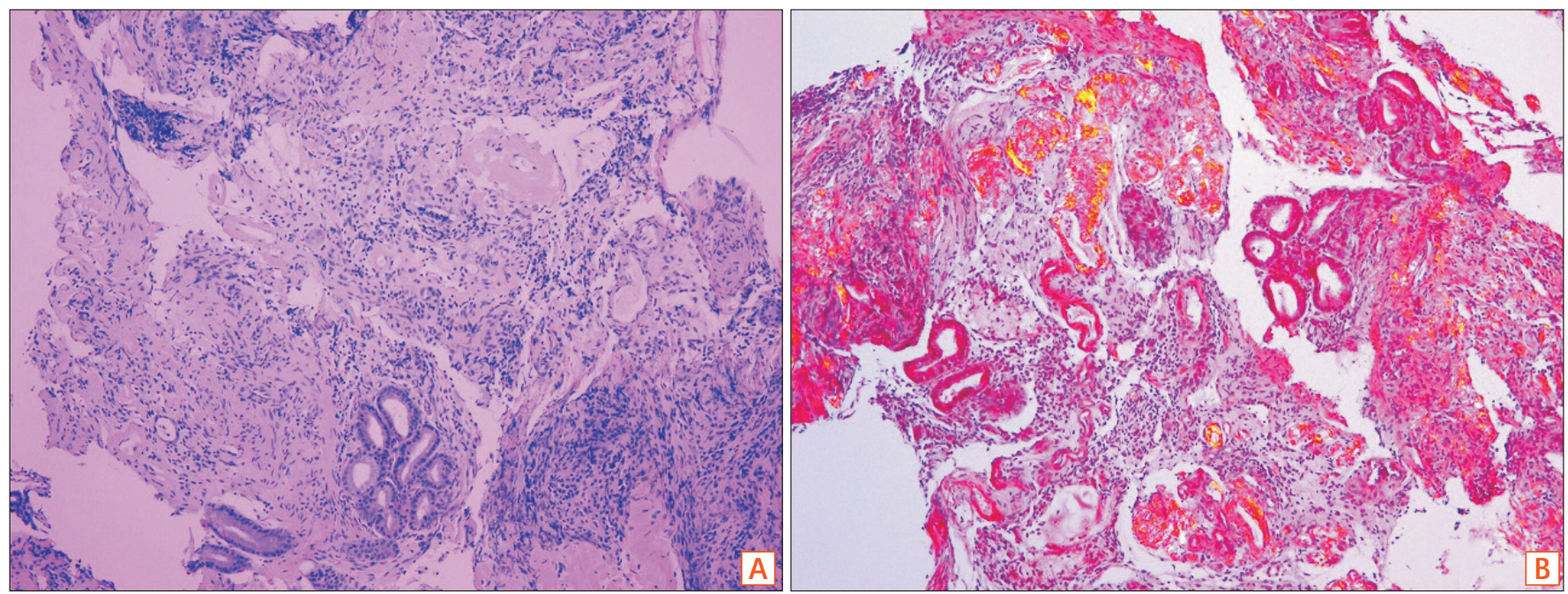

Fig. 2. Histologic findings. (A) Pink, amorphous hyaline materials were present in the mucosa and submucosa of the duodenum (H\&E, $\times 100)$. (B) Polarized light microscopy using Congo red staining indicated the presence of amyloid deposits that exhibited apple-green birefringence $(\times 100)$. 


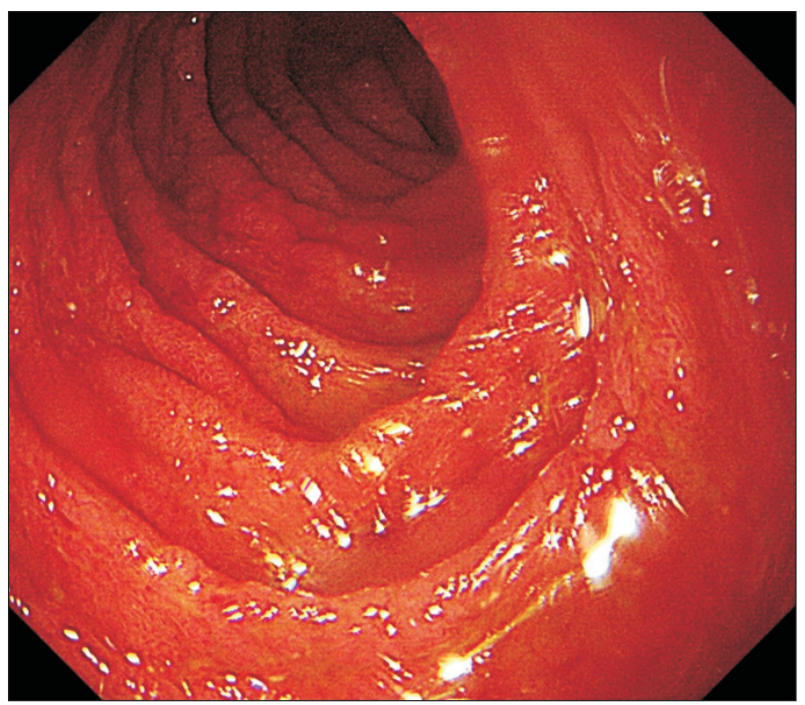

Fig. 3. On follow-up endoscopy, the mucosal edema, erosion, and geographic ulcers of the duodenum showed significant improvement.

terized by extracellular deposition of insoluble polymeric protein fibrils in tissues and organs. There are several types of amyloidosis, and the 2 major forms are AL (primary) and AA (secondary) types. AL amyloidosis is due to the deposition of protein derived from the Ig light chain and is most frequently caused by a clonal expansion of plasma cells. AA amyloidosis results from the deposition of fibrils that are composed of SAA protein, an acute phase reactant. AA amyloidosis may complicate several chronic inflammatory diseases, including IBD. ${ }^{1,2}$ The first report of amyloidosis complicating IBD was published by Oslan and Sussman in 1948. Since the first description, there have been many case reports, but only a few studies, assessing the prevalence and clinical characteristics of amyloidosis complicating IBD due to its rarity. Among IBD patients, AA amyloidosis occurs more frequently in men, who exhibit an approximately 3 -fold greater risk. Furthermore, it occurs more frequently in patients with $\mathrm{CD}$, while amyloidosis secondary to UC is rare. The reported incidence of amyloidosis in IBD patients is approximately $1 \%$ of CD patients and $0.07 \%$ of UC patients. Amyloid nephropathy is the most common manifestation of amyloidosis in association with IBD, while renal involvement is a major contributor to mortality. ${ }^{3,4}$

AA amyloidosis can affect a variety of organ systems, including the heart and kidneys. Kidneys are the most commonly affected organ system. Renal involvement often manifests as asymptomatic proteinuria, nephrotic syndrome, or renal failure., ${ }^{3,4}$ AA amyloidosis also commonly affects the gastrointestinal tract. Gastrointestinal amyloidosis presents as gastrointestinal bleeding, malabsorption, protein-losing enteropathy, or gastrointestinal dysmotility. Nausea, vomiting, or abdominal pain can occur as symptoms of gastrointestinal dysmotility. ${ }^{5,6}$

To diagnose amyloidosis, tissue biopsy is essential to confirm the presence of amyloid protein. Polarized light microscopy using Congo red stain or electron microscopy can be used to confirm such amyloid deposits. Once amyloid is detected, the protein type should be determined by immunohistochemistry, immunoelectron microscopy, or mass spectrometry. Further testing to detect the presence of monoclonal Ig, as well as serum FLC assays, are recommended to distinguish between AL and AA amyloidosis. In the present case, AL amyloidosis was excluded by conducting serum and urine protein electrophoresis, immunofixation electrophoresis, and bone marrow biopsy.

The primary therapy for secondary amyloidosis involves treating the underlying inflammatory or infectious disease. The treatment of underlying diseases also decreases the SAA protein level. Colchicine can be used to treat and prevent secondary amyloidosis, particularly in familial Mediterranean fever. ${ }^{7}$ Anti-proinflammatory cytokines, such as interleukin 6 or tumor necrosis factor antagonists, are effective in some cases of secondary amyloidosis due to rheumatic disorders and hereditary auto-inflammatory disease. ${ }^{8,9}$ There are also fibril-specific agents that interfere with fibril formation. ${ }^{10}$ The management of gastrointestinal amyloidosis is divided into either symptom control or the treatment of the underlying disease. Patients with nausea, vomiting, or abdominal pain due to intestinal dysmotility can be treated with dietary modification, nutritional support, and medications such as prokinetics and antiemetics.

The patient in the present case was previously treated with adalimumab, a tumor necrosis factor inhibitor, which is also used for the treatment of AA amyloidosis. However, the patient required additional therapy for amyloidosis, and glucocorticoids were administered to greatly reduce the severity of the underlying UC. Symptoms of nausea, vomiting, and abdominal pain were resolved, and the CRP level was normalized, within a few days after glucocorticoid treatment. Although glucocorticoid therapy is not an established treatment of gastrointestinal amyloidosis, in some cases, efficacy of steroids and octreotides for protein-losing enteropathy due to gastrointestinal amyloidosis has been reported. ${ }^{11} \mathrm{Glu}$ cocorticoids may modulate inflammation in UC and reduce SAA protein levels. Glucocorticoids also aid in resolution of edema and reduce inflammation in the gastrointestinal tract caused by amyloidosis. The disease course and prognosis of 
the patient will continue to be observed in an outpatient setting.

\section{FINANCIAL SUPPORT}

The authors received no financial support for the research, authorship, and/or publication of this article.

\section{CONFLICT OF INTEREST}

No potential conflict of interest relevant to this article was reported.

\section{AUTHOR CONTRIBUTION}

S.W.P. wrote the case report. S.R.J. provided a critical revision of the manuscript for intellectual content and was responsible for final approval of the manuscript. All authors contributed to writing this case report.

\section{REFERENCES}

1. Lachmann HJ, Goodman HJ, Gilbertson JA, et al. Natural history and outcome in systemic AA amyloidosis. N Engl J Med 2007;356:2361-2371.

2. Tuglular S, Yalcinkaya F, Paydas S, et al. A retrospective analysis for aetiology and clinical findings of 287 secondary amyloidosis cases in Turkey. Nephrol Dial Transplant 2002;17:2003-2005.

3. Greenstein AJ, Sachar DB, Panday AK, et al. Amyloidosis and inflammatory bowel disease: a 50-year experience with 25 patients. Medicine (Baltimore) 1992;71:261-270.
4. Serra I, Oller B, Mañosa M, et al. Systemic amyloidosis in inflammatory bowel disease: retrospective study on its prevalence, clinical presentation, and outcome. J Crohns Colitis 2010;4:269274.

5. Sattianayagam PT, Hawkins PN, Gillmore JD. Systemic amyloidosis and the gastrointestinal tract. Nat Rev Gastroenterol Hepatol 2009;6:608-617.

6. Petre S, Shah IA, Gilani N. Review article: gastrointestinal amyloidosis: clinical features, diagnosis and therapy. Aliment Pharmacol Ther 2008;27:1006-1016.

7. Zemer D, Pras M, Sohar E, Modan M, Cabili S, Gafni J. Colchicine in the prevention and treatment of the amyloidosis of familial Mediterranean fever. N Engl J Med 1986;314:1001-1005.

8. Gottenberg JE, Merle-Vincent F, Bentaberry F, et al. Anti-tumor necrosis factor alpha therapy in fifteen patients with AA amyloidosis secondary to inflammatory arthritides: a followup report of tolerability and efficacy. Arthritis Rheum 2003;48:2019-2024.

9. Miyagawa I, Nakayamada S, Saito K, et al. Study on the safety and efficacy of tocilizumab, an anti-IL-6 receptor antibody, in patients with rheumatoid arthritis complicated with AA amyloidosis. Mod Rheumatol 2014;24:405-409.

10. Dember LM, Hawkins PN, Hazenberg BP, et al. Eprodisate for the treatment of renal disease in AA amyloidosis. N Engl J Med 2007;356:2349-2360.

11. Jeong YS, Jun JB, Kim TH, et al. Successful treatment of proteinlosing enteropathy due to AA amyloidosis with somatostatin analogue and high dose steroid in ankylosing spondylitis. Clin Exp Rheumatol 2000;18:619-621. 